\title{
Product of composition and differentiation operators on a space of entire functions
}

\author{
Pawan Kumar ${ }^{1}$ and Mohd Arief ${ }^{2}$ \\ ${ }^{1}$ Department of Mathematics, Govt. Degree College Kathua, India \\ 2 Department of Mathematics, Central University of Jammu, India
}

Received: 10 May 2018, Accepted: 14 December 2018

Published online: 21 May 2019.

\begin{abstract}
The product of composition operator $C_{\varphi}$ and differentiation operator $D$ is written as $C_{\varphi} D$ and $D C_{\varphi}$ which are defined as $C_{\varphi} D f=f^{\prime} o \varphi$ and $D C_{\varphi} f=(f o \varphi)^{\prime}$ respectively. In this paper, we characterize the continuity of the operators $C_{\varphi} D$ and $D C_{\varphi}$ on $\mathscr{E}$, the space of entire functions.
\end{abstract}

Keywords: Composition operator, differentiation operator, entire functions.

\section{Introduction}

Let $X$ be a non-empty set and $V(X)$ be a vector space of complex valued functions on $X$. If $\varphi: X \rightarrow X$ is a mapping such that $f \circ \varphi \in V(X)$ for all $f \in V(X)$, then the composition transformation $C_{\varphi}: V(X) \rightarrow V(X)$ is defined as

$$
C_{\varphi} f=f o \varphi \forall f \in V(X)
$$

If $V(X)$ is a topological vector space and $C_{\varphi}$ is continuous on $V(X)$, then we call $C_{\varphi}$ as composition operator induced by $\varphi$. Further, let $\psi: X \rightarrow \mathbb{C}$ be a function, then the multiplication transformation $M_{\psi}: V(X) \rightarrow V(X)$ defined as

$$
M_{\psi} f=\psi \cdot f \forall f \in V(X)
$$

If $V(X)$ is a topological vector space and $M_{\psi}$ is continuous on $V(X)$, then $M_{\psi}$ is called the multiplication operator induced by $\psi$. Let $D$ be the differentiation operator defined on $V(X)$ as $D f=f^{\prime}$. The generalized composition operators $C_{\varphi} D$ and $D C_{\varphi}$ on $V(X)$ are defined as $C_{\varphi} D f=f^{\prime} o \varphi$ and $D C_{\varphi} f=(f o \varphi)^{\prime}$ for all $f \in V(X)$ respectively. A complex valued function $f: \mathbb{C} \rightarrow \mathbb{C}$ is called entire function if it is analytic in the whole complex plane $\mathbb{C}$. If $f$ is an entire function, then the power series representation of $\mathrm{f}$ can be written as

$$
f(z)=\sum_{n=0}^{\infty} \hat{f}_{n} z^{n}
$$

where $\left\{\hat{f}_{n}\right\}$ a sequence of complex numbers such that $\lim _{n \rightarrow \infty}\left|\hat{f}_{n}\right|^{\frac{1}{n}}=0$. Conversely on every sequence $\left\{\hat{f}_{n}\right\}$ of complex numbers such that $\lim _{n \rightarrow \infty}\left|\hat{f}_{n}\right|^{\frac{1}{n}}=0$, there is an entire function $f$ represented by (1.1). A metric $d$ on the class of entire functions is defined by $d(f, g)=\sup \left\{\left|\hat{f}_{0}-\hat{g}_{0}\right|,\left|\hat{f}_{n}-\hat{g}_{n}\right|^{\frac{1}{n}}: n \geq 1\right\}$. The class of entire functions topologized by this metric is denoted by $\mathscr{E}$. It has been shown in Iyer [8] that $\mathscr{E}$ is a non-normable complex metrizable locally convex 
topological vector space. In the space $\mathscr{E}$ of entire functions, the convergence of a sequence of entire functions is equivalent to the uniform convergence of entire functions in any circle of finite radius and this convergence is called the strong convergence in $\mathscr{E}$.

The continuous linear functional $F$ on $\mathscr{E}$ is given by $F(f)=\sum_{n=0}^{\infty} f_{n} a_{n}$ where $f(z)=\sum_{n=0}^{\infty} a_{n} z^{n}$ and $\left\{f_{n}\right\}$ be a sequence of complex numbers such that $\left\{\left|\hat{f}_{n}\right|^{\frac{1}{n}}\right\}$ be a bounded sequence. The set of all bounded linear continuous functional on $\mathscr{E}$ is denoted by $\mathscr{E}^{\star}$. For each $n \in \mathbb{N}$, we define $e_{n}: \mathbb{C} \rightarrow \mathbb{C}$ as $e_{n}(z)=z^{n} \forall z \in \mathbb{C}$. Then the sequence $\left\{e_{n}: n \in \mathbb{N}\right\}$ is called a basis for $\mathscr{E}$. A sequence $\left\{\alpha_{n}\right\}$ in $\mathscr{E}$ is called a basis for $\mathscr{E}$ if for each $\alpha \in \mathscr{E}$, there exists a unique sequence $\left\{f_{n}(\alpha)\right\}$ of complex nos such that $\alpha=\sum_{n=0}^{\infty} f_{n}(\alpha) \cdot \alpha_{n}$. For $R>0$, we denote by $\mathbb{D}_{R}$, the open unit disc in $\mathbb{C}$ defined as $\mathbb{D}_{R}=\{z \in \mathbb{C}:|z|<1\}$. The space $\mathscr{E}$ of entire functions has been studied extensively by Iyer $[8,9,10]$ and [11].

In this paper, we initiated the study of generalized composition operators on the space $\mathscr{E}$ of entire functions. Much of the work on composition operators is done on Hardy space. For more about composition operator on Hardy space, we refer to Schwartz [19] and Shapiro [20] .

This paper is organised as follows. In the first section, we give introduction of the work done here. We study the boundedness of the operator $C_{\varphi} D$ in the second section and in the third section, we study the boundedness of the operator $D C_{\varphi}$ on the space $\mathscr{E}$.

\section{Boundedness of the operator $C_{\varphi} D$}

In this section, we shall characterize the boundedness of generalized composition operator $C_{\varphi} D$ on space $\mathscr{E}$ of entire functions. For this purpose, we need the following Lemma.

Lemma 1. Let $f \in \mathscr{E}$. Then for each $z \in \mathbb{D}_{R}$

$$
\left|f^{\prime}(z)\right| \leq \frac{M(R, f) \cdot R}{(R-|z|)^{2}}
$$

Proof. By the Cauchy integral formula for derivative, we have

$$
f^{\prime}(z)=\frac{1}{2 \pi i} \int_{C_{R}} \frac{f(w)}{(w-z)^{2}} d w \text { where } C_{R}:|z|=R
$$

This implies that

$$
\left|f^{\prime}(z)\right| \leq \frac{1}{2 \pi} \int_{C_{R}} \frac{|f(w)|}{|w-z|^{2}}|d w| \leq \frac{M(R, f)}{2 \pi} \frac{1}{(R-|z|)^{2}} \int_{C_{R}}|d w|=\frac{M(R, f)}{2 \pi} \frac{1}{(R-|z|)^{2}} 2 \pi R=\frac{M(R, f) \cdot R}{(R-|z|)^{2}}
$$

Therefore

$$
\left|f^{\prime}(z)\right| \leq \frac{M(R, f) \cdot R}{(R-|z|)^{2}}, \quad \forall z \in \mathbb{D}_{R}
$$

Theorem 1. Let $\varphi: \mathbb{C} \rightarrow \mathbb{C}$ be a mapping and $D: \mathscr{E} \rightarrow \mathscr{E}$ be the differentiation operator. Then the generalized composition operator $C_{\varphi} D: \mathscr{E} \rightarrow \mathscr{E}$ is continuous (bounded) iff $\varphi$ is an entire function.

Proof. Assume that the operator $C_{\varphi} D: \mathscr{E} \rightarrow \mathscr{E}$ is continuous. Then $C_{\varphi} D f=f^{\prime} o \varphi$ is an entire function. In particular for $f=\frac{e_{2}}{2} \in \mathscr{E}$, where $e_{2}(z)=z^{2}$, we have $f^{\prime} o \varphi=e_{1} o \varphi=\varphi$ is an entire function.

Conversely, assume that $\varphi$ is an entire function. In order to prove that $C_{\varphi} D$ is a continuous operator, it is sufficient to show that $C_{\varphi} D$ is continuous at origin. 
Let $R>0$ be given, then $\overline{\mathbb{D}}_{R}$ is a compact subset of $\mathbb{C}$, but $\varphi$ is a continuous map, therefore $\varphi\left(\overline{\mathbb{D}}_{R}\right)$ is compact subset of $\mathbb{C}$ and so we can find $K>M(R, \varphi)$ such that $\varphi\left(\overline{\mathbb{D}}_{R}\right) \subset \mathbb{D}_{K}$. Now, convergence in $\mathscr{E}$ is equivalent to the uniform convergence in any circle of finite radius. Let $\left\{f_{n}\right\}$ be a sequence in $\mathscr{E}$ s.t $f_{n} \rightarrow 0$. Then for each $\varepsilon>0$, there exists $n_{0} \in N$ such that $M\left(K, f_{n}\right)<\varepsilon \cdot \frac{K_{0}^{2}}{K}$ for $n \geq n_{0}$, where $K_{0}=K-M(K, \varphi)$.

From Lemma 1, we have

$$
\left|f_{n}^{\prime}(\varphi(z))\right| \leq \frac{M\left(K, f_{n}\right) K}{(K-|\varphi(z)|)^{2}} \leq \frac{M\left(K, f_{n}\right) K}{(K-M(K, \varphi))^{2}}<\varepsilon, \quad \forall z \in \mathbb{D}_{R}, \quad n \geq n_{0}
$$

Therefore

$$
C_{\varphi} D f_{n}=f_{n}^{\prime} o \varphi \rightarrow 0 \text { as } n \rightarrow \infty
$$

This proves that the operator $C_{\varphi} D$ is continuous.

Theorem 2. Let $T \in C(\mathscr{E})$. Then $T$ is a generalized composition operator of the type $C_{\varphi} D$ for some entire function $\varphi: \mathbb{C} \rightarrow \mathbb{C}$ iff

$$
T e_{n}=n\left[T \frac{e_{2}}{2}\right]^{n-1}
$$

Proof. Suppose, there exists an entire function $\varphi: \mathbb{C} \rightarrow \mathbb{C}$ such that $T=C_{\varphi} D$. Now

$$
T e_{n}=C_{\varphi} D e_{n}=e_{n}^{\prime} o \varphi=n \varphi^{n-1}=n\left[e_{1} o \varphi\right]^{n-1}=n\left[C_{\varphi} D \frac{e_{2}}{2}\right]^{n-1}=n\left[T\left(\frac{e_{2}}{2}\right)\right]^{n-1}, \quad \forall n \in \mathbb{N}
$$

Conversely, assume that $T e_{n}=n\left[T\left(\frac{e_{2}}{2}\right)\right]^{n-1}$.

Setting $T\left(\frac{e_{2}}{2}\right)=\varphi$, then $\varphi$ is an entire function and so $C_{\varphi} D$ is a generalized composition operator. Now

$$
\begin{aligned}
T f & =T\left[\sum_{n=0}^{\infty} \hat{f}_{n} e_{n}\right]=\sum_{n=0}^{\infty} \hat{f}_{n} T e_{n}=\sum_{n=0}^{\infty} \hat{f}_{n} \cdot n\left[T\left(\frac{e_{2}}{2}\right)\right]^{n-1}=\sum_{n=0}^{\infty} \hat{f}_{n} \cdot n \varphi^{n-1}=\sum_{n=0}^{\infty} \hat{f}_{n} \cdot e_{n}^{\prime} o \varphi=\sum_{n=0}^{\infty} \hat{f}_{n} \cdot C_{\varphi} D e_{n} \\
& =C_{\varphi} D\left[\sum_{n=0}^{\infty} \hat{f}_{n} e_{n}\right]=C_{\varphi} D f, \quad \forall f \in \mathscr{E}
\end{aligned}
$$

Therefore, $T=C_{\varphi} D$ and so $T$ is a generalized composition operator.

Theorem 3. Let $T \in C(\mathscr{E})$. Then $T$ is generalized composition operator of the type $C_{\varphi} D$ iff $T^{\star} A \subset B$, where $A=\left\{E_{z}\right.$ : $z \in \mathbb{C}\}$ and $B=\left\{E_{z} o D: z \in \mathbb{C}\right\}$

Proof. Firstly, suppose that $\mathrm{T}$ be a generalized composition operator. Then $\exists$ an entire function $\varphi: \mathbb{C} \rightarrow \mathbb{C}$ such that $T=C_{\varphi} D$.

Now, for $z \in \mathbb{C}, E_{z} \in \mathscr{E}^{\star}$, we have

$$
\begin{aligned}
\left(T^{\star} E_{z}\right) f & =E_{z}(T f)=E_{z}\left(C_{\varphi} D f\right)=E_{z}\left(f^{\prime} o \varphi\right)=\left(f^{\prime} o \varphi\right)(z)=f^{\prime}(\varphi(z))=E_{\varphi(z)} f^{\prime}=\left(E_{\varphi(z)} o D\right) f, \text { for all } f \in \mathscr{E} \text { and } z \in \mathbb{C} . \\
& \Rightarrow T^{\star} E_{z} f=\left(E_{\varphi(z)} o D\right) f=\left(E_{w} o D\right) f, \text { where } \varphi: \mathbb{C} \rightarrow \mathbb{C} \text { is defined by } \varphi(z)=w .
\end{aligned}
$$

Hence $T^{\star} E_{z}=E_{w} o D$, for some $w \in \mathbb{C}$.

$$
\therefore T^{\star} A \subset B \text {. }
$$


Conversely, suppose that $T^{\star} A \subset B$, where $A=\left\{E_{z}: z \in \mathbb{C}\right\}$ and $B=\left\{E_{z} o D: z \in \mathbb{C}\right\}$. Now for $f \in \mathscr{E}$ and $z \in \mathbb{C}$, we have

$$
(T f)(z)=E_{z}(T f)=\left(T^{\star} E_{z}\right) f=\left(E_{w} o D\right) f \text { for some } w \in \mathbb{C}
$$

Now define $\varphi: \mathbb{C} \rightarrow \mathbb{C}$ as $\varphi(z)=w$. Then

$$
(T f)(z) E_{\varphi(z)} f^{\prime}=f^{\prime}(\varphi(z))=\left(C_{\varphi} D f\right)(z)
$$

This implies that $T=C_{\varphi} D$. Hence $\mathrm{T}$ is a generalized composition operator.

Theorem 4. Let $T=C_{\varphi} D \in C(\mathscr{E})$. Then $T^{\star}: \mathscr{E}^{\star} \rightarrow \mathscr{E}^{\star}$ is a generalized composition operator if $\varphi(z)=\alpha z$.

Proof. Let $F \in \mathscr{E}^{\star}, f \in \mathscr{E}$ and $\varphi(z)=\alpha z$. Define $\psi: \mathbb{C} \rightarrow \mathbb{C}$ by $\psi(z)=\alpha z$. Then

$$
F(z)=\sum_{n=0}^{\infty} F_{n} z^{n}, \quad f(z)=\sum_{n=0}^{\infty} \hat{f}_{n} z^{n}
$$

Therefore

$$
F^{\prime}(z)=\sum_{n=1}^{\infty} n F_{n} z^{n-1}, \quad f^{\prime}(z)=\sum_{n=1}^{\infty} n \hat{f}_{n} z^{n-1}
$$

Now

$$
\left(f^{\prime} o \varphi\right)(z)=\sum_{n=0}^{\infty}\left(\widehat{f^{\prime} o \varphi}\right)(n) \cdot z^{n}=\sum_{n=1}^{\infty}\left(\widehat{f^{\prime} o \varphi}\right)(n-1) z^{n-1}
$$

and

$$
\left(f^{\prime} o \varphi\right)(z)=f^{\prime}(\varphi(z))=\sum_{n=1}^{\infty} n \hat{f}_{n}(\varphi(z))^{n-1}=\sum_{n=1}^{\infty} n \hat{f}_{n} \alpha^{n-1} z^{n-1}
$$

From (1) and (2), we get

$$
\left(\widehat{f^{\prime} o \varphi}\right)(n-1)=n \hat{f}_{n} \alpha^{n-1}=n z^{n-1} \alpha^{n-1} \text { where } \hat{f}_{n}=z^{n-1}
$$

Also

$$
F^{\prime}(\psi(z))=\sum_{n=1}^{\infty} n F_{n}(\psi(z))^{n-1}=\sum_{n=1}^{\infty} n F_{n} \alpha^{n-1} z^{n-1}
$$

Now

$$
\begin{aligned}
\left(C_{\varphi} D^{\star} F\right)(f) & =F\left[C_{\varphi} D f\right]=F\left(f^{\prime} o \varphi\right)=\sum_{n=0}^{\infty} F_{n}\left(f^{\prime} o \varphi\right)(n) \\
& =F_{0}\left(f^{\prime} o \varphi\right)(0)+\sum_{n=1}^{\infty} F_{n}\left(f^{\prime} o \varphi\right)(n-1) \quad\left[\because F_{0}\left(f^{\prime} o \varphi\right)(0)=0\right] \\
& =\sum_{n=1}^{\infty} n F_{n} \cdot \hat{f}_{n} \alpha^{n-1}=F^{\prime}(\psi(f))=\left(C_{\psi} D F\right)(f) .
\end{aligned}
$$

Therefore $C_{\varphi} D^{\star}=C_{\psi} D$ for some entire function $\psi$. 


\section{Boundedness of the operator $D C_{\varphi}$.}

In this section, we characterize the boundedness of the generalized composition operators $D C_{\varphi}$ on the space $\mathscr{E}$ of entire functions.

Theorem 5. Let $\varphi: \mathbb{C} \rightarrow \mathbb{C}$ be a mapping such that $\varphi^{\prime}$ is bounded and $D: \mathscr{E} \rightarrow \mathscr{E}$ be the differentiation operator. Then the generalized composition operator $D C_{\varphi}: \mathscr{E} \rightarrow \mathscr{E}$ is continuous iff $\varphi^{\prime}$ is constant.

Proof. Suppose that the operator $D C_{\varphi}: \mathscr{E} \rightarrow \mathscr{E}$ is continuous. Then $D C_{\varphi} f=(f \circ \varphi)^{\prime}$ is entire for all $f \in \mathscr{E}$.

In particular for $f=z \in \mathscr{E}$, we have $D C_{\varphi} f=f^{\prime}(\varphi) \cdot \varphi^{\prime}=1 . \varphi^{\prime}=\varphi^{\prime}$ is an entire function. Therefore $\varphi^{\prime}$ being a bounded entire function must be constant.

Conversely, suppose that $\varphi^{\prime}$ is constant. Then $\varphi$ is differentiable and hence continuous. To prove that $D C_{\varphi}$ is continuous in $\mathscr{E}$, it is enough to prove that $D C_{\varphi}$ is continuous at origin. Let $R>0$ be given, then $\overline{\mathbb{D}}_{R}$ is a compact subset of $\mathbb{C}$, but $\varphi$ is a continuous map, therefore $\varphi\left(\overline{\mathbb{D}}_{R}\right)$ is compact subset of $\mathbb{C}$ and so we can find $K>M(R, \varphi)$ such that $\varphi\left(\overline{\mathbb{D}}_{R}\right) \subset \mathbb{D}_{K}$. Now, convergence in $\mathscr{E}$ is equivalent to the uniform convergence in any circle of finite radius.

Let $\left\{f_{n}\right\}$ be a sequence in $\mathscr{E}$ s.t $f_{n} \rightarrow 0$. Then for each $\varepsilon>0$, there exists $n_{0} \in N$ such that $M\left(K, f_{n}\right)<\varepsilon \cdot \frac{K_{0}^{2}}{K\left|\varphi^{\prime}(z)\right|}$, where $K_{0}=K-M(K, \varphi)$ for $n \geq n_{0}$.

From Lemma (1), we have

$$
\left|f_{n}^{\prime}(\varphi(z)) \cdot \varphi^{\prime}(z)\right|=\left|f_{n}^{\prime}(\varphi(z))\right| \cdot\left|\varphi^{\prime}(z)\right| \leq \frac{K M\left(K, f_{n}\right) \cdot\left|\varphi^{\prime}(z)\right|}{(K-|\varphi(z)|)^{2}}<\varepsilon, \quad \forall z \in \mathbb{D}_{R}, \quad n \geq n_{0} .
$$

Hence $D C_{\varphi} f_{n}=\left(f_{n} o \varphi\right)^{\prime} \rightarrow 0$ as $n \rightarrow \infty$.

Theorem 6. Let $T \in C(\mathscr{E})$. Then $T$ be a generalized composition operator of type $D C_{\varphi}$ iff

$$
T e_{n}=T e_{1}^{n} \text { for } n=0,1,2,3 \ldots
$$

Proof. Let $\mathrm{T}$ be a generalized composition operator of the type $D C_{\varphi}$. Then $\exists$ an entire function $\varphi: \mathbb{C} \rightarrow \mathbb{C}$ such that $T=D C_{\varphi}$. Now

$$
T e_{n}=D C_{\varphi} e_{n}=\left(e_{n} o \varphi\right)^{\prime}=\left[\varphi^{n}\right]^{\prime}=\left[\left(e_{1} o \varphi\right)^{n}\right]^{\prime}=\left[e_{1}^{n} o \varphi\right]^{\prime}=D C_{\varphi} e_{1}^{n}=T e_{1}^{n} \text { for } n=0,1,2,3, \ldots
$$

Conversely, suppose that $T e_{n}=T e_{1}^{n}$. Then set $T e_{1}^{n}=\left(\varphi^{n}\right)^{\prime}$. Clearly $\varphi$ is an entire function. Now

$$
\begin{aligned}
T f & =T\left[\sum_{n=0}^{\infty} \hat{f}_{n} e_{n}\right]=\sum_{n=0}^{\infty} \hat{f}_{n} T e_{n}=\sum_{n=0}^{\infty} \hat{f}_{n} T e_{1}^{n}=\sum_{n=0}^{\infty} \hat{f}_{n}\left(\varphi^{n}\right)^{\prime}=\sum_{n=0}^{\infty} \hat{f}_{n}\left(e_{n} o \varphi\right)^{\prime}=\sum_{n=0}^{\infty} \hat{f}_{n} D C_{\varphi} e_{n} \\
& =D C_{\varphi}\left[\sum_{n=0}^{\infty} \hat{f}_{n} e_{n}\right]=\left(D C_{\varphi}\right) f, \quad \text { for every } f \in \mathscr{E} .
\end{aligned}
$$

Therefore $T=D C_{\varphi}$ and so $T$ be generalized composition operator.

Theorem 7. Let $T \in C(\mathscr{E})$. Then $T$ be a generalized composition operator of the type $D C_{\varphi}$ iff $T^{\star} A \subset B$, where $A=\left\{E_{z}\right.$ : $z \in \mathbb{C}\}$ and $B=\left\{E_{w} D C_{\varphi}: w \in \mathbb{C}\right.$ and $\varphi$ an entire function $\}$ 
Proof. First suppose that $T \in C(\mathscr{E})$ be a generalized composition operator. Then $\exists$ an entire function $\varphi: \mathbb{C} \rightarrow \mathbb{C}$ such that $T=D C_{\varphi}$. Now

$$
\left(T^{\star} E_{z}\right) f=E_{z}(T f)=E_{z}\left(D C_{\varphi} f\right)=E_{z}(f o \varphi)^{\prime}=\left(E_{z} D\right)(f o \varphi)=\left(E_{z} D\right)\left(C_{\varphi} f\right)=\left(E_{z} D C_{\varphi}\right) f
$$

Thus $T^{\star} A \subset B$.

Conversely, suppose that $T^{\star} A \subset B$. Now for $f \in \mathscr{E}$ and $z \in \mathbb{C}$, we have

$$
(T f)(z)=E_{z}(T f)=T^{\star}\left(E_{z} f\right)=\left(T^{\star} E_{z}\right)(f)=\left(E_{w} D C_{\varphi_{1}}\right)(f)
$$

where $w \in \mathbb{C}$ and $\varphi_{1}$ an entire function. Now define $\varphi_{2}: \mathbb{C} \rightarrow \mathbb{C}$ as $\varphi_{2}(z)=w$. Then

$$
\begin{aligned}
(T f)(z) & =\left(E_{\varphi_{2}(z)} D C_{\varphi_{1}}\right)(f)=E_{\varphi_{2}(z)}\left(D C_{\varphi_{1}} f\right)=\left(D C_{\varphi_{1}} f\right)\left(\varphi_{2}(z)\right)=\left(D f o \varphi_{1} o \varphi_{2}\right)(z) \\
& =D(f o \varphi)(z), \text { where } \varphi=\varphi_{1} o \varphi_{2} \text { is an entire function } . \\
& =\left(D C_{\varphi} f\right)(z) \Rightarrow T=D C_{\varphi}
\end{aligned}
$$

This completes the proof.

Theorem 8. Let $T=D C_{\varphi} \in C(\mathscr{E})$. Then $T^{\star}: \mathscr{E}^{\star} \rightarrow \mathscr{E}^{\star}$ be a generalized composition operator if $\varphi(z)=z$.

Proof. Let $\varphi: \mathbb{C} \rightarrow \mathbb{C}$ is defined by $\varphi(z)=z$. Now, let $F \in \mathscr{E}^{\star}, f \in \mathscr{E}$. Then we have

$$
F(z)=\sum_{n=0}^{\infty} F_{n} z^{n}, \quad f(z)=\sum_{n=0}^{\infty} \hat{f}_{n} z^{n}, \quad F^{\prime}(z)=\sum_{n=1}^{\infty} n F_{n} z^{n-1}, \quad f^{\prime}(z)=\sum_{n=1}^{\infty} n \hat{f}_{n} z^{n-1}
$$

Define $\psi: \mathbb{C} \rightarrow \mathbb{C}$ by $\psi(z)=z$. Then clearly $\psi$ is an entire function. Now

$$
\begin{aligned}
& (f \circ \varphi)^{\prime}(z)=f^{\prime}(\varphi(z)) \varphi^{\prime}(z)=\sum_{n=1}^{\infty} n \hat{f}_{n}(\varphi(z))^{n-1} 1=\sum_{n=1}^{\infty} n \hat{f}_{n} z^{n-1} \text { and } \\
& (f \circ \varphi)^{\prime}(z)=\sum_{n=1}^{\infty}(\widehat{f o \varphi})^{\prime}(n) z^{n}=\sum_{n=1}^{\infty}(\widehat{f o \varphi})^{\prime}(n-1) z^{n-1}
\end{aligned}
$$

Since $(f o \varphi)^{\prime}(z)$ has a unique representation. Therefore, we have

$$
(\widehat{f o \varphi})^{\prime}(n-1)=n \hat{f}_{n}=n z^{n-1}, \text { where } \hat{f}_{n}=z^{n-1}
$$

Also, we have

$$
F^{\prime}(\psi(z)) \psi^{\prime}(z)=\sum_{n=1}^{\infty} n F_{n}(\psi(z))^{n-1} 1=\sum_{n=1}^{\infty} n F_{n} z^{n-1}
$$

Now

$$
\begin{aligned}
\left(T^{\star} F\right)(f) & =F(T f)=F\left(D C_{\varphi} f\right)=F(f o \varphi)^{\prime}=\sum_{n=0}^{\infty}(\widehat{f o \varphi})^{\prime}(n) F_{n}=\sum_{n=1}^{\infty}(\widehat{f o \varphi})^{\prime}(n-1) F_{n}=\sum_{n=1}^{\infty} n \cdot F_{n} \cdot z^{n-1} \\
& =F^{\prime}(\psi(z)) \psi^{\prime}(z)=\left(D C_{\varphi} F\right)(f)=(T F)(f) .
\end{aligned}
$$

Therefore, $T^{\star}=T$ and so $T^{\star}$ be a generalized composition operator. 


\section{Competing interests}

The authors declare that they have no competing interests.

\section{Authors' contributions}

All authors have contributed to all parts of the article. All authors read and approved the final manuscript.

\section{References}

[1] Boyd D. M., Composition operators on $H^{p}(A)$, Pacific J. Math. 62 (1976), 55-60.

[2] Conway J. B., A course in functional analysis, Springer Verlag, 1985.

[3] Cowen C. C., Composition operator on $H^{2}$, J. Oper. Theory 9 (1983), 77-106.

[4] Cowen C. C. and MacCluear B., Composition operators on spaces of analytic functions, Stud. Adv. Math., CRC Press. Boca Ration, 1995.

[5] Duren P., Theory of $H^{p}$ spaces, Academic Press New York, 1973.

[6] Garnett J. B., Bounded analytic functions, Revised first edition. Graduate Texts in Mathematics, 236.Springer, New York,2007.

[7] Hibschweiler R. A. and Portnoy N., Composition Operators followed by differentiation between Bergman and Hardy spaces, Rocky Mountain J.Math.35, 843-855 (2005).

[8] Iyer V. G., On the space of integral functions-I, J. Indian Math. Soc. 12 (1948).

[9] Iyer V. G., On the space of integral functions-II, Quart J. Math. Oxford Ser (2) Vol. I (1950), 86-96.

[10] Iyer V. G., On the space of integral functions-Ill, Proc. Amer. Math. Soc. Vol. 3 (1952), 874-883.

[11] Iyer V. G., On the space of integral functions-IV, Proc. Amer. Math. Soc. 7 (1956), 644-649.

[12] Kumar Pawan and Sharma S. D., Weighted composition operators from weighted Bergman-Nevanlinna spaces to zygmund spaces, Int.J.Mod. Math.Sci.3(1),(2012),31-54.

[13] Kumar Pawan and Sharma S. D., Generalized composition operators from weighted Bergman-Nevanlinna spaces to zygmund spaces, Int.J.Mod. Math.Sci.1(3),(2012),160-162.

[14] Kumar Pawan and Abbas Zaheer, Product of multiplication and composition operators on weighted Hardy spaces , S.S International Journal of Pure and Applied Mathematics Vol.1,Issue 2,2015.

[15] Kumar Pawan and Abbas Zaheer, Composition operator between weighted Hardy type spaces, International journal of pure and Applied mathematics Vol.106,No.3,2016.

[16] Kumar Pawan and Abbas Zaheer, Product of multiplication composition and differentation operators on weighted Hadry space , International Journal of computational and applied mathematics,Vol.12,No.3,2017.

[17] Li S. and Stevic S., Composition followed by differentiation between $H^{\infty}$ and $\alpha$-Bloch spaces, Houston J. Math. 35(2009), $327-$ 340.

[18] Ohno S., Product of composition and differentiation between Hardy spaces, Bull Austral Math. Soc.73(2006), 235-243.

[19] Schwartz H. J., Composition operators on $H^{p}$, Thesis University of Toledo, 1969.

[20] Shapiro J. H., Composition operators and classical function theory, Springer-Verlag New York 1993.

[21] Singh R. K. and Komal B. S., Composition operators on lp and its adjoint, Proc. Amer. Math. Soc. 70 (1978), 21-25.

[22] Shield A. L., Weighted shift operator and analytic function theory, in topic in operator theory, Math surveys, No 13, Amer. Math. soc. Providence 1947.

[23] Sharma S. D. and Kumar R., Substitution Operators on Hardy -Orlicz spaces, Proc.Nat.Acad.Sci.India Sect.A61 (1991),535-541.

[24] Sharma A. K., Product of composition Multiplication and differentiation between Bergman and Bloch type spaces, Turkish. J. Math. 34 (2010), 117.

[25] Sharma A. K., Volterra composition operators between Bergman Nevanlinna and Bloch-type spaces, Demonstratio Mathematica VolXLII No. 32009.

[26] Sharma A. K. and Sharma S. D., Weighted composition operators between Bergman type spaces, Comm. Korean Math. Soc. 21 No. 3(2006), 465-474. 
[27] Swantan D. W., Composition operators on $H^{p}(D)$, Ph. D. Thesis North western University, 1974.

[28] Zhu K., Operator theory in function spaces, Marcel-Dekker, New York 1990.

[29] Zhu K., Spaces of holomorphic functions in the unit ball. Graduate Text in Mathematics,vol.226. Springer,New York (2005).

[30] Zhu X., Weighted composition operators from area Nevanlinna spaces into Bloch spaces, Applied Mathematics and computation, 215 (2010), 4340-4346. 